\title{
On a Relative Version of Fujita's Freeness Conjecture
}

\author{
Yujiro Kawamata
}

June 29, 2021

\section{Introduction}

The following is Fujita's freeness conjecture:

Conjecture 1.1. Let $X$ be a smooth projective variety of dimension $n$ and $H$ an ample divisor. Then the invertible sheaf $\mathcal{O}_{X}\left(K_{X}+m H\right)$ is generated by global sections if $m \geq n+1$, or $m=n$ and $H^{n} \geq 2$.

We have a stronger local version of Conjecture 1.1 (cf. [4]):

Conjecture 1.2. Let $X$ be a smooth projective variety of dimension $n, L$ a nef and big invertible sheaf on $X$, and $x \in X$ a point. Assume that $L^{n}>n^{n}$ and $L^{d} Z \geq n^{d}$ for any irreducible subvariety $Z$ of $X$ of dimension $d$ which contains $x$. Then the natural homomorphism

$$
H^{0}\left(X, \omega_{X} \otimes L\right) \rightarrow \omega_{X} \otimes L \otimes \kappa(x)
$$

is surjective.

We shall extend the above conjecture to a relative setting.

Let $f: Y \rightarrow X$ be a surjective morphism of smooth projective varieties. We note that the geometric fibers of $f$ are not necessarily connected. Assume that there exists a normal crossing divisor $B=\sum_{i=1}^{h} B_{i}$ on $X$ such that $f$ is smooth over $X_{0}=X \backslash B$. Then the sheaves $R^{q} f_{*} \omega_{Y / X}$ are locally free for $q \geq 0$ ([2] for $q=0$ and [6] in general). We note that even if we change the birational model of $Y$, the sheaf $R^{q} f_{*} \omega_{Y / X}$ does not change.

The relative version is the following: 
Conjecture 1.3. Let $f: Y \rightarrow X$ be a surjective morphism from a smooth projective variety to a smooth projective variety of dimension $n$ such that $f$ is smooth over $X_{0}=X \backslash B$ for a normal crossing divisor $B$ on $X$. Let $H$ be an ample divisor on $X$. Then the locally free sheaf $R^{q} f_{*} \omega_{Y} \otimes \mathcal{O}_{X}(m H)$ is generated by global sections if $m \geq n+1$, or $m=n$ and $H^{n} \geq 2$.

We have again a stronger local version:

Conjecture 1.4. Let $f: Y \rightarrow X$ be a surjective morphism from a smooth projective variety to a smooth projective variety of dimension $n$ such that $f$ is smooth over $X_{0}=X \backslash B$ for a normal crossing divisor $B$ on $X$. Let $L$ be a nef and big invertible sheaf on $X$, and $x \in X$ a point. Assume that $L^{n}>n^{n}$ and $L^{d} Z \geq n^{d}$ for any irreducible subvariety $Z$ of $X$ of dimension $d$ which contains $x$. Then the natural homomorphism

$$
H^{0}\left(X, R^{q} f_{*} \omega_{Y} \otimes L\right) \rightarrow R^{q} f_{*} \omega_{Y} \otimes L \otimes \kappa(x)
$$

is surjective for any $q \geq 0$.

In [4], the following strategy toward Conjectures 1.1] and 1.2 was developed:

Theorem 1.5 (44). Let $X$ be a smooth projective variety of dimension $n$, $L$ a nef and big invertible sheaf, and $x \in X$ a point. Assume the followig condition: for any effective $\mathbb{Q}$-divisor $D_{0}$ on $X$ such that $\left(X, D_{0}\right)$ is $K L T$, there exists an effective $\mathbb{Q}$-divisor $D$ on $X$ such that

(1) $D \equiv \lambda L$ for some $0<\lambda<1$,

(2) The pair $\left(X, D_{0}+D\right)$ is properly log canonical at $x$, and

(3) $\{x\}$ is a log canonical center for $\left(X, D_{0}+D\right)$.

Then the natural homomorphism

$$
H^{0}\left(X, \omega_{X} \otimes L\right) \rightarrow \omega_{X} \otimes L \otimes \kappa(x)
$$

is surjective.

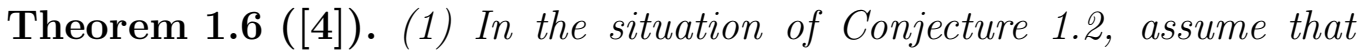
$\operatorname{dim} X \leq 3$. Then the condition of Theorem 1.5 is satisfied for $L$.

(2) In the situation of Conjecture [1.1, assume that $\operatorname{dim} X=4$. Then the condition of Theorem 1.5 is satisfied for $L=m H$ with $m \geq 5$ or $m=4$ and $H^{4} \geq 2$. 
Our main result is the relative version of Theorem 1.5:

Theorem 1.7. Let $f: Y \rightarrow X$ be a surjective morphism from a smooth projective variety to a smooth projective variety of dimension $n$ such that $f$ is smooth over $X_{0}=X \backslash B$ for a normal crossing divisor $B$ on $X$. Let $L$ be a nef and big invertible sheaf on $X$, and $x \in X$ a point. Assume the followig condition: for any effective $\mathbb{Q}$-divisor $D_{0}$ on $X$ such that $\left(X, D_{0}\right)$ is $K L T$, there exists an effective $\mathbb{Q}$-divisor $D$ on $X$ such that

(1) $D \equiv \lambda L$ for some $0<\lambda<1$,

(2) The pair $\left(X, D_{0}+D\right)$ is properly log canonical at $x$, and

(3) $\{x\}$ is a log canonical center for $\left(X, D_{0}+D\right)$.

Then the natural homomorphism

$$
H^{0}\left(X, R^{q} f_{*} \omega_{Y} \otimes L\right) \rightarrow R^{q} f_{*} \omega_{Y} \otimes L \otimes \kappa(x)
$$

is surjective for any $q \geq 0$. In particular, Conjecture 1.4 for $\operatorname{dim} X \leq 3$ and Conjecture 1.3 for $\operatorname{dim} X=4$ hold.

The main tool for the proof of Theorem 1.5 was the $\mathbb{Q}$-divisor version of the Kodaira vanishing theorem, so-called Kawamata-Viehweg vanishing theorem. In order to prove Theorem 1.7, we shall extend the Kollár vanishing theorem, the relative version of the Kodaira vanishing theorem, to the $\mathbb{Q}$ divisor version. We had a similar result already in [3] Theorem 3.3, but we need more precise version.

In $\S 2$, we review the construction of the canonical extension for a variation of Hodge structures. In order to describe the behavior of the Hodge bundles at infinity, we shall introduce the notion of parabolic structures over arbitrary dimensional base in $\S 3$. In $\S 4$, we shall prove the base change theorem of the parabolic structures (Lemma 4.1) and derive the correct $\mathbb{Q}$-divisor version of the Kollár vanishing theorem (Theorem 4.2). The main result will be proved in $\S 5$.

Remark 1.8. (1) It is easy to prove Conjecture 1.1 in the case $L$ is very ample. [6] proved Conjecture 1.3 in the case $L$ is very ample.

(2) Let $\pi: P=\mathbb{P}(\mathcal{F}) \rightarrow X$ be the projective space bundle associated to our locally free sheaf $\mathcal{F}=R^{q} f_{*} \omega_{Y / X}$, and $\mathcal{O}_{P}(H)$ the tautological invertible sheaf. Then the sheaf $\mathcal{F} \otimes \mathcal{O}_{X}\left(K_{X}+L\right)$ is generated by global sections, if and only if $\mathcal{O}_{P}\left(H+\pi^{*}\left(K_{X}+L\right)\right)$ is so. Since $K_{P}=-r H+\pi^{*}\left(K_{X}+\operatorname{det}(\mathcal{F})\right)$ if $r=\operatorname{rank}(\mathcal{F})$, Conjecture 1.3 is related to, but different from, Conjecture 1.1. 


\section{Review on the Hodge bundles}

Let $X$ be a smooth projective variety, and $B$ a normal crossing divisor. Let $H_{\mathbb{Z}}$ be a local system on $X_{0}=X \backslash B$. A variation of Hodge structures on $X_{0}$ is defined as a decreasing filtration $\left\{\mathcal{F}_{0}^{p}\right\}$ of locally free subsheaves on $\mathcal{H}_{0}=H_{\mathbb{Z}} \otimes \mathcal{O}_{X_{0}}$ which satisfy certain axioms ([1]). Assume in addition that all the local monodromies of $H_{\mathbb{Z}}$ around the branches of $B$ are unipotent. Then we define a locally free sheaf $\mathcal{H}$ on $X$ called the canonical extension of $\mathcal{H}_{0}$ as follows. Let $\left\{z_{1}, \cdots, z_{n}\right\}$ be local coordinates at a given point $x \in X$ such that $B$ is defined by an equation $z_{1} \cdots z_{r}$ near $x$. Let $T_{i}$ be the monodromies of $H_{\mathbb{Z}}$ around the branches of $B$ defined by $z_{i}=0$. Let $v$ be a multi-valued flat section of $H_{\mathbb{Z}}$. Then the expression

$$
s=\exp \left(-\frac{1}{2 \pi \sqrt{-1}} \sum_{i=1}^{r} \log T_{i} \log z_{i}\right) v
$$

is single-valued and gives a holomorphic section of $\mathcal{H}_{0}$, where $\log T_{i}$ is defined by a finite power series of $T_{i}-1$. The canonical extension $\mathcal{H}$ is defined as a locally free sheaf on $X$ whose local basis near $x$ consists of the $s$ when $v$ making a basis of $H_{\mathbb{Z}}$. The filtration $\left\{\mathcal{F}_{0}^{p}\right\}$ extends to a filtration $\left\{\mathcal{F}^{p}\right\}$ of locally free subsheaves on $\mathcal{H}$ such that the quotients $\mathcal{H} / \mathcal{F}^{p}$ are also locally free for all $p(\mathbb{8})$.

Let $f: Y \rightarrow X$ be a surjective morphism from another smooth projective variety which is smooth over $X_{0}$. Let $d=\operatorname{dim} Y-\operatorname{dim} X, Y_{0}=f^{-1}\left(X_{0}\right)$ and $f_{0}=\left.f\right|_{Y_{0}}$. Then $H_{\mathbb{Z}}=R^{d+q} f_{0 *} \mathbb{Z}_{Y_{0}}$ is a variation of Hodge structures on $X_{0}$ for any $q \geq 0$. We know that the canonical extension $\mathcal{F}^{d}$ in this case coincides with the direct image sheaf $R^{q} f_{*} \omega_{Y / X}$ ([2] and [6]).

The following lemma is obvious:

Lemma 2.1. Let $X$ be a smooth projective variety, and $B$ a normal crossing divisor. Let $H_{\mathbb{Z}}$ be a variation of Hodge structures on $X_{0}=X \backslash B$ whose local monodromies around the branches of $B$ are unipotent, and $\mathcal{H}$ the canonical extension of $\mathcal{H}_{0}=H_{\mathbb{Z}} \otimes \mathcal{O}_{X_{0}}$ on $X$. Let $\pi: X^{\prime} \rightarrow X$ be a generically finite and surjective morphism from a smoth projective variety such that $B^{\prime}=\left(\pi^{*} B\right)_{\text {red }}$ is a normal crossing divisor. Let $H_{\mathbb{Z}}^{\prime}=\pi^{*} H_{\mathbb{Z}}$ be the induced variation of Hodge structures on $X_{0}^{\prime}=X^{\prime} \backslash B^{\prime}$, and $\mathcal{H}^{\prime}$ the canonical extension of $\mathcal{H}_{0}^{\prime}=$ $H_{\mathbb{Z}}^{\prime} \otimes \mathcal{O}_{X_{0}^{\prime}}$ on $X^{\prime}$. Then $\mathcal{H}^{\prime}=\pi^{*} \mathcal{H}$. 


\section{Parabolic structure in several variables}

We generalize the notion of parabolic structures on vector bundles ([7]) over higher dimensional base space:

Definition 3.1. Let $f: Y \rightarrow X$ be a surjective morphism of smooth projective varieties. Assume that there exists a normal crossing divisor $B=$ $\sum_{i=1}^{h} B_{i}$ on $X$ such that $f$ is smooth over $X_{0}=X \backslash B$. Fixing a nonnegative integer $q$, we define a parabolic structure on the sheaf $\mathcal{F}=R^{q} f_{*} \omega_{Y / X}$. It is a decreasing filtration of subsheaves $F^{t_{1}, \ldots, t_{h}}=F^{t_{1}, \ldots, t_{h}}(\mathcal{F}) \subset \mathcal{F}$ with multi-indices $t=\left(t_{1}, \ldots, t_{h}\right)\left(t_{i} \in \mathbb{R}_{\geq 0}\right)$ defined by

$$
\Gamma\left(U, F^{t_{1}, \ldots, t_{h}}(\mathcal{F})\right)=\left\{s \in \Gamma(U, \mathcal{F}) \mid\left(\prod_{i} z_{i}^{-t_{i}}\right) s \text { is } L^{2}\right.
$$

with respect to the Hodge metric\},

where $z_{i}$ is a local equation of the branch $B_{i}$ on an open subset $U \subset X$.

Lemma 3.2. (1) $F^{t} \supset F^{t^{\prime}}$ for $t \leq t^{\prime}$, i.e., $t_{i} \leq t_{i}^{\prime}$ for all $i$.

(2) $F^{t_{1}, \ldots, t_{i}+\epsilon, \ldots, t_{h}}=F^{t_{1}, \ldots, t_{i}, \ldots, t_{h}}$ for $0<\epsilon \ll 1$.

(3) $F^{t_{1}, \ldots, t_{i}+1, \ldots, t_{h}}=F^{t_{1}, \ldots, t_{i}, \ldots, t_{h}} \otimes \mathcal{O}_{X}\left(-B_{i}\right)$.

(4) Let $Y_{0}=f^{-1}\left(X_{0}\right), f_{0}=\left.f\right|_{Y_{0}}$ and $d=\operatorname{dim} Y-\operatorname{dim} X$. If all the local monodromies of $R^{d+q} f_{0 *} \mathbb{Z}_{Y_{0}}$ around the branches of $B$ are unipotent, then $F^{t}=F^{0}$ for any $t=\left(t_{1}, \ldots, t_{h}\right)$ with $0 \leq t_{i}<1$.

Proof. (1) through (3) are obvious. (4) follows from the fact that the growth of the Hodge metric is logarithmic in this case $([2])$.

Remark 3.3. For negative values of the $t_{i}$, we can also define $F^{t}$ as subsheaves of $\mathcal{F} \otimes \mathcal{O}_{X}(m B)$ for sufficiently large $m$ by using Lemma 3.2 (3). We shall also write $F^{\sum_{i} t_{i} B_{i}}$ instead of $F^{t_{1}, \ldots, t_{h}}$.

Definition 3.4. For a local section $s \in \Gamma(U, \mathcal{F})$, we define its order of growth along $B$ by

$$
\operatorname{ord}(s)=\sum_{i} \operatorname{ord}_{i}(s) B_{i}=\inf \left\{\sum_{i}\left(1-t_{i}\right) B_{i} \mid s \in \Gamma\left(U, F^{t_{1}, \ldots, t_{h}}(\mathcal{F})\right)\right\} .
$$

We note that $s \notin \Gamma\left(U, F^{B-\operatorname{ord}(s)}(\mathcal{F})\right)$, and

$$
\Gamma\left(U, F^{t_{1}, \ldots, t_{h}}(\mathcal{F})\right)=\left\{s \in \Gamma(U, \mathcal{F}) \mid \operatorname{ord}(s)+\sum_{i} t_{i} B_{i}<B\right\} .
$$


There is a nice local basis of the sheaf $\mathcal{F}=R^{q} f_{*} \omega_{Y / X}$ :

Lemma 3.5. At any point $x \in X$, there exists an open neighborhood $U$ in the classical topology and a $\Gamma\left(U, \mathcal{O}_{U}\right)$-free basis $\left\{s_{1}, \ldots, s_{k}\right\}$ of $\Gamma(U, \mathcal{F})$ such that

$$
\left(\prod_{i} z_{i}^{\left\llcorner t_{i}+\operatorname{ord}_{i}\left(s_{1}\right)\right\lrcorner}\right) s_{1}, \ldots,\left(\prod_{i} z_{i}^{\left\llcorner t_{i}+\operatorname{ord}_{i}\left(s_{k}\right)\right\lrcorner}\right) s_{k}
$$

generates $\Gamma\left(U, F^{t_{1}, \ldots, t_{h}}(\mathcal{F})\right)$ for any $t$, where the $z_{i}$ are local equations of the $B_{i}$ on $U$. In particular, the sheaf $F^{t_{1}, \ldots, t_{h}}(\mathcal{F})$ is locally free for any $t$.

Proof. We shall prove that the filtration $F^{t}$ is determined by the local monodromies of the cohomology sheaf $R^{d+q} f_{0 *} \mathbb{Z}_{Y_{0}}$ around the branches of $B$ which are known to be quasi-unipotent, where $d=\operatorname{dim} Y-\operatorname{dim} X$.

We take an open neighborhood $U$ of $x \in X$ in the classical topology which is isomorphic to a polydisk with coordinates $\left\{z_{1}, \ldots, z_{n}\right\}$ such that $B \cap U$ is defined by $z_{1} \cdots z_{r}=0$. To simplify the notation, we write $X$ instead of $U$. There exists a finite surjective and Galois morphism $\pi: X^{\prime} \rightarrow X$ from a smooth variety which is etale over $X_{0}$ such that, for the induced morphism $f^{\prime}: Y^{\prime} \rightarrow X^{\prime}$ from a desingularization $Y^{\prime}$ of the fiber product $Y \times_{X} X^{\prime}$, the local system $R^{d+q} f_{0 *}^{\prime} \mathbb{Z}_{Y_{0}^{\prime}}$ has unipotent local monodromies around the branches of $B^{\prime}=\pi^{-1}(B)$, where we set $X_{0}^{\prime}=\pi^{-1}\left(X_{0}\right), Y_{0}^{\prime}=f^{\prime-1}\left(X_{0}^{\prime}\right)$ and $f_{0}^{\prime}=\left.f^{\prime}\right|_{Y_{0}^{\prime}}$. Let $\sigma: Y^{\prime} \rightarrow Y$ be the induced morphism.

We may assume that $X^{\prime}$ is isomorphic to a polydisk centered at a point $x^{\prime}=\pi^{-1}(x)$ with coordinates $\left\{z_{1}^{\prime}, \ldots, z_{n}^{\prime}\right\}$, and the morphism $\pi: X^{\prime} \rightarrow X$ is given by $\pi^{*} z_{i}=z_{i}^{\prime m_{i}}$ for some positive integers $m_{i}$, where $m_{i}=1$ for $i>r$. The Galois group $G=\operatorname{Gal}\left(X^{\prime} / X\right)$ is isomorphic to $\prod_{i} \mathbb{Z} /\left(m_{i}\right)$. Let $g_{1}, \ldots, g_{r}$ be generators of $G$ such that $g_{i}^{*} z_{j}^{\prime}=\zeta_{m_{i}}^{\delta_{i j}} z_{j}^{\prime}$ for some roots of unity $\zeta_{m_{i}}$ of order $m_{i}$.

The group $G$ acts on the sheaves $R^{q} f_{*}^{\prime} \omega_{Y^{\prime} / X^{\prime}}$ and $\omega_{X^{\prime}}$ equivariantly such that the invariant part $\left(\pi_{*}\left(R^{q} f_{*}^{\prime} \omega_{Y^{\prime} / X^{\prime}} \otimes \omega_{X^{\prime}}\right)\right)^{G}$ is isomorphic to $R^{q} f_{*} \omega_{Y / X} \otimes$ $\omega_{X}$, because $\left(\sigma_{*} \omega_{Y^{\prime}}\right)^{G}=\omega_{Y}$ and $R^{p} \sigma_{*} \omega_{Y^{\prime}}=0$ for $p>0$.

The vector space $R^{q} f_{*}^{\prime} \omega_{Y^{\prime} / X^{\prime}} \otimes \kappa\left(x^{\prime}\right)$ is decomposed into simultaneous eigenspaces with respect to the action of $G$. Let $s_{x^{\prime}}$ be a simultaneous eigenvector such that $g_{i}^{*} s_{x^{\prime}}=\zeta_{m_{i}}^{a_{i}} s_{x^{\prime}}$ for some $a_{i}$ with $0 \leq a_{i}<m_{i}$. Let $\bar{s}^{\prime}$ be a section of $R^{q} f_{*}^{\prime} \omega_{Y^{\prime} / X^{\prime}}$ which extends $s_{x^{\prime}}$. Then the section

$$
s^{\prime}=\frac{1}{\prod_{i} m_{i}} \sum_{i=1}^{r} \sum_{k_{i}=0}^{m_{i}-1} \frac{\left(\prod_{i} g_{i}^{k_{i}}\right)^{*} \bar{s}^{\prime}}{\prod_{i} \zeta_{m_{i}}^{a_{i} k_{i}}}
$$


satisfies that $s^{\prime}\left(x^{\prime}\right)=s_{x^{\prime}}$ and $g_{i}^{*} s^{\prime}=\zeta_{m_{i}}^{a_{i}} s^{\prime}$.

On the other hand, $d z_{1}^{\prime} \wedge \cdots \wedge d z_{n}^{\prime}=\left(\prod_{i} m_{i}^{-1} z_{i}^{\prime 1-m_{i}}\right) d z_{1} \wedge \cdots \wedge d z_{n}$ is a generating section of $\omega_{X^{\prime}}$. Therefore, $\left(\prod_{i} z_{i}^{\prime-a_{i}}\right) s^{\prime}$ descends to a section $s$ of $R^{q} f_{*} \omega_{Y / X}$. If the $s_{x^{\prime}}$ varies among a basis of $R^{q} f_{*}^{\prime} \omega_{Y^{\prime} / X^{\prime}} \otimes \kappa\left(x^{\prime}\right)$, then the corresponding sections $s$ make a basis of the locally free sheaf $R^{q} f_{*} \omega_{Y / X}$.

We have $\operatorname{ord}_{i}(s)=a_{i} / m_{i}$, since the Hodge metric on the sheaf $R^{q} f_{*}^{\prime} \omega_{Y^{\prime} / X^{\prime}}$ has logarithmic growth along $B^{\prime}$. Therefore, the sections $\left(\prod_{i} z_{i}^{\left\llcorner t_{i}+a_{i} / m_{i}\right\lrcorner}\right) s$ form a basis of a locally free sheaf $F^{t_{1}, \ldots, t_{h}}\left(R^{q} f_{*} \omega_{Y / X}\right)$.

Remark 3.6. The Hodge metric and the flat metric on the canonical extension of the variation of Hodge structures $R^{d+q} f_{0 *} \mathbb{Z}_{Y_{0}} \otimes \mathcal{O}_{X_{0}}$ coincide when restricted to the subsheaf $R^{q} f_{*} \omega_{Y / X}$. Therefore, the statement that the Hodge metric on the canonical extension has logarithmic growth is easily proved for the sheaf $R^{q} f_{*} \omega_{Y / X}$.

\section{Base change and a relative vanishing theo- rem}

By using the basis obtained in Lemma 3.5, we can study the base change property of the sheaf $R^{q} f_{*} \omega_{Y / X}$ :

Lemma 4.1. Let $\pi: X^{\prime} \rightarrow X$ be a generically finite and surjective morphism from a smooth projective variety such that $B^{\prime}=\left(\pi^{*} B\right)_{\mathrm{red}}=\sum_{i^{\prime}=1}^{h^{\prime}} B_{i^{\prime}}^{\prime}$ is a normal crossing divisor. Let $\mu: Y^{\prime} \rightarrow Y \times_{X} X^{\prime}$ be a birational morphism from a smooth projective variety such that the induced morphism $f^{\prime}: Y^{\prime} \rightarrow X^{\prime}$ is smooth over $X_{0}^{\prime}=X^{\prime} \backslash B^{\prime}$. Let $\sigma: Y^{\prime} \rightarrow Y$ be the induced morphism. Then the following hold.

(1) Let $\left\{s_{1}, \ldots, s_{k}\right\}$ be the basis of $\Gamma\left(U, R^{q} f_{*} \omega_{Y / X}\right)$ in Lemma 3.5, and let $U^{\prime}$ be an open subset of $X^{\prime}$ in the classical topology such that $\pi\left(U^{\prime}\right) \subset U$. Then the equality $\operatorname{ord}\left(\pi^{*} s_{j}\right)=\pi^{*} \operatorname{ord}\left(s_{j}\right)$ holds, and the basis $\left\{\pi^{*} s_{1}, \ldots, \pi^{*} s_{k}\right\}$ of $\Gamma\left(U^{\prime}, \pi^{*} R^{q} f_{*} \omega_{Y / X}\right)$ satisfies the conclusion of Lemma 3.5 in the sense that sections

$$
\left(\prod_{i^{\prime}} z_{i^{\prime}}^{\prime\left\llcorner t_{i^{\prime}}+\operatorname{ord}_{i^{\prime}}\left(\pi^{*} s_{1}\right)\right\lrcorner}\right) \pi^{*} s_{1}, \ldots,\left(\prod_{i^{\prime}} z_{i^{\prime}}^{\prime\left\llcorner t_{i^{\prime}}+\operatorname{ord}_{i^{\prime}}\left(\pi^{*} s_{k}\right)\right\lrcorner}\right) \pi^{*} s_{k}
$$


form a basis of $\Gamma\left(U^{\prime}, F^{t_{1}^{\prime}, \ldots, t_{h^{\prime}}^{\prime}}\left(R^{q} f_{*}^{\prime} \omega_{Y^{\prime} / X^{\prime}}\right)\right)$ for any $t^{\prime}=\left(t_{1}^{\prime}, \ldots, t_{h^{\prime}}^{\prime}\right)$, where the $z_{i^{\prime}}^{\prime}$ are local equations of the $B_{i^{\prime}}^{\prime}$ on $U^{\prime}$. In particular, the sections

$$
\left(\prod_{i^{\prime}} z_{i^{\prime}}^{\prime\left\llcorner\operatorname{ord}_{i^{\prime}}\left(\pi^{*} s_{1}\right)\right\lrcorner}\right) \pi^{*} s_{1}, \ldots,\left(\prod_{i^{\prime}} z_{i^{\prime}}^{\prime\left\llcorner\operatorname{ord}_{i^{\prime}}\left(\pi^{*} s_{k}\right)\right\lrcorner}\right) \pi^{*} s_{k} .
$$

form a basis of $\Gamma\left(U^{\prime}, R^{q} f_{*}^{\prime} \omega_{Y^{\prime} / X^{\prime}}\right)$.

(2) There is an equality of subsheaves of $\pi^{*} R^{q} f_{*} \omega_{Y / X}$ :

$$
\begin{aligned}
& F_{1}^{t_{1}^{\prime}, \ldots, t_{h^{\prime}}^{\prime}}\left(R^{q} f_{*}^{\prime} \omega_{Y^{\prime} / X^{\prime}}\right) \\
& =\sum_{t} \pi^{*} F^{t_{1}, \ldots, t_{h}}\left(R^{q} f_{*} \omega_{Y / X}\right) \otimes \mathcal{O}_{X^{\prime}}\left(-\left\llcorner\sum_{i^{\prime}} t_{i^{\prime}}^{\prime} B_{i^{\prime}}^{\prime}+\sum_{i}\left(1-t_{i}\right) \pi^{*} B_{i}\right\lrcorner\right) .
\end{aligned}
$$

In particular,

$$
R^{q} f_{*}^{\prime} \omega_{Y^{\prime} / X^{\prime}}=\sum_{t} \pi^{*} F^{t_{1}, \ldots, t_{h}}\left(R^{q} f_{*} \omega_{Y / X}\right) \otimes \mathcal{O}_{X^{\prime}}\left(-\left\llcorner\sum_{i}\left(1-t_{i}\right) \pi^{*} B_{i}\right\lrcorner\right) .
$$

Proof. (1) Since the $s_{j}$ are derived from the basis in the case of unipotent monodromies, we obtain our assertion by Lemma 2.1.

(2) We can check the assertion locally. We write $\pi^{*} B_{i}=\sum_{i^{\prime}} m_{i i^{\prime}} B_{i^{\prime}}$ for some nonnegative integers $m_{i i^{\prime}}$. Then the left hand side is generated by the sections

$$
\left.\left(\prod_{i^{\prime}} z_{i^{\prime}}^{\prime\left\llcorner t^{\prime}\right.}{ }^{\prime} \operatorname{ord}_{i^{\prime}}\left(\pi^{*} s_{j}\right)\right\lrcorner\right) \pi^{*} s_{j}
$$

for $1 \leq j \leq k$, while each component of the right hand side is by

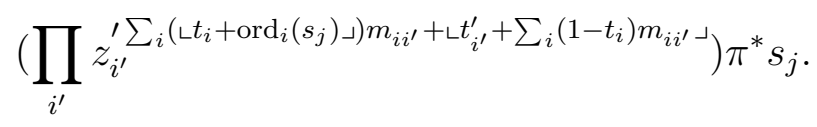

Since $\operatorname{ord}_{i^{\prime}}\left(\pi^{*} s_{j}\right)=\sum_{i} \operatorname{ord}_{i}\left(s_{j}\right) m_{i i^{\prime}}$, we should compare

$$
\left\llcorner t_{i^{\prime}}^{\prime}+\sum_{i} \operatorname{ord}_{i}\left(s_{j}\right) m_{i i^{\prime}}\right\lrcorner
$$

and

$$
\min _{t}\left\{\sum_{i}\left(\left\llcorner t_{i}+\operatorname{ord}_{i}\left(s_{j}\right)\right\lrcorner\right) m_{i i^{\prime}}+\left\llcorner t_{i^{\prime}}^{\prime}+\sum_{i}\left(1-t_{i}\right) m_{i i^{\prime}}\right\lrcorner\right\}
$$


We have

$$
\begin{aligned}
& \sum_{i}\left(\left\llcorner t_{i}+\operatorname{ord}_{i}\left(s_{j}\right)\right\lrcorner\right) m_{i i^{\prime}}+\left\llcorner t_{i^{\prime}}^{\prime}+\sum_{i}\left(1-t_{i}\right) m_{i i^{\prime}}\right\lrcorner-\left\llcorner t_{i^{\prime}}^{\prime}+\sum_{i} \operatorname{ord}_{i}\left(s_{j}\right) m_{i i^{\prime}}\right\lrcorner \\
& >\sum_{i}\left(\left\llcorner t_{i}+\operatorname{ord}_{i}\left(s_{j}\right)\right\lrcorner\right) m_{i i^{\prime}}+\sum_{i}\left(1-t_{i}-\operatorname{ord}_{i}\left(s_{j}\right)\right) m_{i i^{\prime}}-1>-1,
\end{aligned}
$$

hence

$$
\begin{aligned}
& \min _{t}\left\{\sum_{i}\left(\left\llcorner t_{i}+\operatorname{ord}_{i}\left(s_{j}\right)\right\lrcorner\right) m_{i i^{\prime}}+\left\llcorner t_{i^{\prime}}^{\prime}+\sum_{i}\left(1-t_{i}\right) m_{i i^{\prime}}\right\lrcorner\right\} \\
& \geq\left\llcorner t_{i^{\prime}}^{\prime}+\sum_{i} \operatorname{ord}_{i}\left(s_{j}\right) m_{i i^{\prime}}\right\lrcorner .
\end{aligned}
$$

On the other hand, if we set $t_{i}=1-\operatorname{ord}_{i}\left(s_{j}\right)-\epsilon_{i}$ for $0<\epsilon_{i} \ll 1$, then

$$
\left.\sum_{i}\left(\left\llcorner t_{i}+\operatorname{ord}_{i}\left(s_{j}\right)\right\lrcorner\right) m_{i i^{\prime}}+\left\llcorner t_{i^{\prime}}^{\prime}+\sum_{i}\left(1-t_{i}\right) m_{i i^{\prime}}\right\lrcorner\right\}=\left\llcorner t_{i^{\prime}}^{\prime}+\sum_{i} \operatorname{ord}_{i}\left(s_{j}\right) m_{i i^{\prime}}\right\lrcorner .
$$

Therefore, they are equal. Since the minimum is attained at a value of $t$ which does not depend on $i^{\prime}$ but only on $j$, we obtain the equality.

Now we state the vanishing theorem for $\mathbb{Q}$-divisors in the relative setting. This theorem will be used as an essential tool in the proof of the main theorem.

Theorem 4.2. In the situation of Definition 3.1, let $L$ be a nef and big $\mathbb{Q}$-divisor on $X$ whose fractional part is supported on $B$. Then

$$
H^{p}\left(X, \sum_{t} F^{t_{1}, \ldots, t_{h}}\left(R^{q} f_{*} \omega_{Y / X}\right) \otimes \omega_{X}\left(\left\ulcorner L-\sum_{i}\left(1-t_{i}\right) B_{i}\right\urcorner\right)\right)=0
$$

for $p>0$ and $q \geq 0$, where the sum is taken inside the sheaf $R^{q} f_{*} \omega_{Y} \otimes$ $\mathcal{O}_{X}(\ulcorner L\urcorner)$.

Proof. By [2], there exists a normal crossing divisor $\bar{B}$ such that $B \leq \bar{B}$ which satisfies the following: there exists a finite surjective and Galois morphism $\pi: X^{\prime} \rightarrow X$ from a smooth projective variety which is etale over $\bar{X}_{0}=X \backslash \bar{B}$ and such that $\pi^{*} L$ has integral coefficients and all the local monodromies of $R^{d+q} f_{0 *}^{\prime} \mathbb{Z}_{Y_{0}^{\prime}}$ are unipotent under the notation of Lemma 4.1. We replace $B$ by $\bar{B}$ and let $G$ be the Galois group of $\pi$. By [6] Theorem 2.1 and [3] Theorem 3.3 , we have

$$
H^{p}\left(X^{\prime}, R^{q} f_{*}^{\prime} \omega_{Y^{\prime}} \otimes \mathcal{O}_{X^{\prime}}\left(\pi^{*} L\right)\right)=0
$$


for $p>0$. Since $\pi^{*}\left(K_{X}+B\right)=K_{X^{\prime}}+B^{\prime}$, we have by Lemma 4.1

$$
\begin{aligned}
& H^{p}\left(X^{\prime}, \sum_{t} \pi^{*} F^{t_{1}, \ldots, t_{h}}\left(R^{q} f_{*} \omega_{Y / X}\right)\right. \\
& \left.\quad \otimes \mathcal{O}_{X^{\prime}}\left(-\left\llcorner\sum_{i^{\prime}} B_{i^{\prime}}^{\prime}+\sum_{i}\left(1-t_{i}\right) \pi^{*} B_{i}\right\lrcorner+\pi^{*}\left(K_{X}+B+L\right)\right)\right)=0 .
\end{aligned}
$$

We want to calculate the $G$-invariant part of the locally free sheaf

$$
\begin{aligned}
\mathcal{G}=F^{t_{1}, \ldots, t_{h}} & \left(R^{q} f_{*} \omega_{Y / X}\right) \\
& \otimes \pi_{*}\left(\mathcal{O}_{X^{\prime}}\left(-\left\llcorner\sum_{i^{\prime}} B_{i^{\prime}}^{\prime}+\sum_{i}\left(1-t_{i}\right) \pi^{*} B_{i}\right\lrcorner+\pi^{*}\left(K_{X}+B+L\right)\right)\right) .
\end{aligned}
$$

For this purpose, let $A$ be the largest divisor on $X$ such that

$$
\pi^{*} A \leq\left\ulcorner-\sum_{i^{\prime}} B_{i^{\prime}}^{\prime}-\sum_{i}\left(1-t_{i}\right) \pi^{*} B_{i}\right\urcorner+\pi^{*}(B+L) .
$$

This is equivalent to the condition

$$
\pi^{*} A<\pi^{*}\left(L-\sum_{i}\left(1-t_{i}\right) B_{i}\right)+\pi^{*} B .
$$

Hence we obtain

$$
A=\left\ulcorner L-\sum_{i}\left(1-t_{i}\right) B_{i}\right\urcorner
$$

Since

$$
0=H^{p}(X, \mathcal{G})^{G}=H^{p}\left(X, \sum_{t} F^{t_{1}, \ldots, t_{h}}\left(R^{q} f_{*} \omega_{Y / X}\right) \otimes \omega_{X}(A)\right)
$$

our assertion is proved.

Remark 4.3. We note that the sum

$$
\sum_{t} F^{t_{1}, \ldots, t_{h}}\left(R^{q} f_{*} \omega_{Y / X}\right) \otimes \omega_{X}\left(\left\ulcorner L-\sum_{i}\left(1-t_{i}\right) B_{i}\right\urcorner\right)
$$

is a locally free sheaf because it is the $G$-invariant part of a locally free sheaf as shown in the above proof, though the expression looks complicated. It coincides with the subsheaf of $L^{2}$-sections of the locally free sheaf $R^{q} f_{*} \omega_{Y / X} \otimes$ $\omega_{X}(\ulcorner L\urcorner)$. We can consider the non-vanishing problem for this sheaf. 


\section{Proof of Theorem 1.7}

Let $\left\{s_{1}, \cdots, s_{k}\right\}$ be the basis of $R^{q} f_{*} \omega_{Y / X}$ on a neighborhood $U$ of $x$ which is obtained in Lemma 3.5. We shall prove that the image of the homomorphism

$$
H^{0}\left(X, R^{q} f_{*} \omega_{Y} \otimes L\right) \rightarrow R^{q} f_{*} \omega_{Y} \otimes L \otimes \kappa(x)
$$

contains $s_{j} \otimes \omega_{X} \otimes L \otimes \kappa(x)$ for any $j$. Let us consider ord $\left(s_{j}\right)$ as an effective $\mathbb{Q}$-divisor on $X$ by setting the coefficients of the irreducible components of $B$ which do not intersect $U$ to be 0 . By the assumption of the theorem, there exists an effective $\mathbb{Q}$-divisor $D$ such that $D \sim_{\mathbb{Q}} \lambda L$ with $0<\lambda<1$, $\left(X, \operatorname{ord}\left(s_{j}\right)+D\right)$ is properly $\log$ canonical at $x$, and that $\{x\}$ is a minimal $\log$ canonical center. By the perturbation of $D$, we may assume that $\{x\}$ is the only $\log$ canonical center which contains $x$, and there exists only one log canonical place $E$ above the center $\{x\}$. Let $\mu: X^{\prime} \rightarrow X$ be a birational morphism from a smooth projective variety such that $E$ appears as a smooth divisor on $X^{\prime}$. We write

$$
\mu^{*}\left(K_{X}+\operatorname{ord}\left(s_{j}\right)+D\right)=K_{X^{\prime}}+E+F
$$

where the coefficients of $F$ are less than 1 . We may assume that the union of the exceptional locus of $\mu$ and the support of $\mu^{-1}(B+D)$ is a normal crossing divisor. Let $B^{\prime}=\mu^{*} B_{\text {red }}=\sum_{i^{\prime}} B_{i^{\prime}}^{\prime}$. Since

$$
K_{X^{\prime}}+(1-\lambda) \mu^{*} L=\mu^{*}\left(K_{X}+L\right)-E-F+\mu^{*} \operatorname{ord}\left(s_{j}\right)
$$

we obtain by Theorem 4.2

$$
\begin{aligned}
& H^{1}\left(X^{\prime}, \sum_{t^{\prime}} F^{t_{1}^{\prime}, \ldots, t_{h^{\prime}}^{\prime}}\left(R^{q} f_{*}^{\prime} \omega_{Y^{\prime} / X^{\prime}}\right) \otimes \mathcal{O}_{X^{\prime}}\left(\mu^{*}\left(K_{X}+L\right)\right.\right. \\
& \left.\left.\quad-E+\left\ulcorner-F+\mu^{*} \operatorname{ord}\left(s_{j}\right)-\sum_{i^{\prime}}\left(1-t_{i^{\prime}}^{\prime}\right) B_{i^{\prime}}^{\prime}\right\urcorner\right)\right)=0 .
\end{aligned}
$$

Since

$$
\begin{aligned}
& \sum_{t^{\prime}} F^{t_{1}^{\prime}, \ldots, t_{h^{\prime}}^{\prime}}\left(R^{q} f_{*}^{\prime} \omega_{Y^{\prime} / X^{\prime}}\right) \otimes \mathcal{O}_{X^{\prime}}\left(\mu^{*}\left(K_{X}+L\right)\right. \\
& \left.\left.+\left\ulcorner-F+\mu^{*} \operatorname{ord}\left(s_{j}\right)-\sum_{i^{\prime}}\left(1-t_{i^{\prime}}^{\prime}\right) B_{i^{\prime}}^{\prime}\right\urcorner\right)\right)
\end{aligned}
$$


is a locally free sheaf on $X^{\prime}$, we have a surjective homomorphism

$$
\begin{aligned}
& H^{0}\left(X^{\prime}, \sum_{t^{\prime}} F_{1}^{t_{1}^{\prime}, \ldots, t_{h^{\prime}}^{\prime}}\left(R^{q} f_{*}^{\prime} \omega_{Y^{\prime} / X^{\prime}}\right) \otimes \mathcal{O}_{X^{\prime}}\left(\mu^{*}\left(K_{X}+L\right)\right.\right. \\
& \left.\left.+\left\ulcorner-F+\mu^{*} \operatorname{ord}\left(s_{j}\right)-\sum_{i^{\prime}}\left(1-t_{i^{\prime}}^{\prime}\right) B_{i^{\prime}}^{\prime}\right\urcorner\right)\right) \\
& \rightarrow H^{0}\left(E, \sum_{t^{\prime}} F^{t_{1}^{\prime}, \ldots, t_{h^{\prime}}^{\prime}}\left(R^{q} f_{*}^{\prime} \omega_{Y^{\prime} / X^{\prime}}\right) \otimes \mathcal{O}_{E}\left(\mu^{*}\left(K_{X}+L\right)\right.\right. \\
& \left.\left.+\left\ulcorner-F+\mu^{*} \operatorname{ord}\left(s_{j}\right)-\sum_{i^{\prime}}\left(1-t_{i^{\prime}}^{\prime}\right) B_{i^{\prime}}^{\prime}\right\urcorner\right)\right) .
\end{aligned}
$$

We have

$$
\mu_{*}\left(\left\ulcorner-F+\mu^{*} \operatorname{ord}\left(s_{j}\right)-\sum_{i^{\prime}}\left(1-t_{i^{\prime}}^{\prime}\right) B_{i^{\prime}}^{\prime}\right\urcorner\right) \leq 0
$$

if $0 \leq t_{i^{\prime}}^{\prime}<1$. Hence

$$
\begin{aligned}
& H^{0}\left(X^{\prime}, \sum_{t^{\prime}} F^{t_{1}^{\prime}, \ldots, t_{h^{\prime}}^{\prime}}\left(R^{q} f_{*}^{\prime} \omega_{Y^{\prime} / X^{\prime}}\right) \otimes \mathcal{O}_{X^{\prime}}\left(\mu^{*}\left(K_{X}+L\right)\right.\right. \\
& \left.\left.\quad+\left\ulcorner-F+\mu^{*} \operatorname{ord}\left(s_{j}\right)-\sum_{i^{\prime}}\left(1-t_{i^{\prime}}^{\prime}\right) B_{i^{\prime}}^{\prime}\right\urcorner\right)\right) \\
& \subset H^{0}\left(X, R^{q} f_{*} \omega_{Y / X} \otimes \mathcal{O}_{X}\left(K_{X}+L\right)\right) .
\end{aligned}
$$

We note that the $t_{i^{\prime}}^{\prime}$ need not be contained in the interval $[0,1)$ in the above sum. On the other hand, if we define the $t_{i^{\prime}}^{\prime}$ by

$$
\sum_{i^{\prime}} t_{i^{\prime}}^{\prime} B_{i^{\prime}}^{\prime}=\sum_{i^{\prime}}\left(1-\epsilon_{i^{\prime}}^{\prime}\right) B_{i^{\prime}}^{\prime}-\mu^{*} \operatorname{ord}\left(s_{j}\right)
$$

for sufficiently small and positive numbers $\epsilon_{i^{\prime}}^{\prime}$, then the divisor

$$
\left\ulcorner-F+\mu^{*} \operatorname{ord}\left(s_{j}\right)-\sum_{i^{\prime}}\left(1-t_{i^{\prime}}^{\prime}\right) B_{i^{\prime}}^{\prime}\right\urcorner
$$

is effective and its support does not contain $E$, even if $E$ is contained in $B^{\prime}$. Since $\operatorname{ord}\left(\mu^{*} s_{j}\right)=\mu^{*} \operatorname{ord}\left(s_{j}\right)$, we have $\mu^{*} s_{j} \in F^{t_{1}^{\prime}, \ldots, t_{h^{\prime}}^{\prime}}\left(R^{q} f_{*}^{\prime} \omega_{Y^{\prime} / X^{\prime}}\right)$ for such $t_{i^{\prime}}^{\prime}$. Hence

$$
\begin{aligned}
& \mu^{*} s_{j} \otimes \mathcal{O}_{E}\left(\mu^{*}\left(K_{X}+L\right)\right) \\
& \in H^{0}\left(E, \sum_{t^{\prime}} F^{t_{1}^{\prime}, \ldots, t_{h^{\prime}}^{\prime}}\left(R^{q} f_{*}^{\prime} \omega_{Y^{\prime} / X^{\prime}}\right) \otimes \mathcal{O}_{E}\left(\mu^{*}\left(K_{X}+L\right)\right.\right. \\
& \left.\left.\quad+\left\ulcorner-F+\mu^{*} \operatorname{ord}\left(s_{j}\right)-\sum_{i^{\prime}}\left(1-t_{i^{\prime}}^{\prime}\right) B_{i^{\prime}}^{\prime}\right\urcorner\right)\right) .
\end{aligned}
$$

Therefore, $s_{j} \otimes \omega_{X} \otimes L \otimes \kappa(x)$ is contained in the image of $H^{0}\left(X, R^{q} f_{*} \omega_{Y} \otimes L\right)$. 


\section{References}

[1] P. Griffiths. Period of integrals on algebraic manifolds III. Publ. Math. I.H.E.S. 38(1970), 125-180.

[2] Y. Kawamata. Characterization of abelian varieties. Compositio Math. 43(1981), 253-276.

[3] Y. Kawamata. Pluricanonical systems on minimal algebraic varieties. Invent. Math. 79 (1985), 567-588.

[4] Y. Kawamata. On Fujita's freeness conjecture for 3-folds and 4folds, alg-geom/9510004, Math. Ann. 308 (1997), 491-505.

[5] Y. Kawamata, K. Matsuda and K. Matsuki. Introduction to the minimal model problem. Adv. St. Pure Math. 10(1987), 283-360.

[6] J. Kollár. Higher direct images of dualizing sheaves I. Ann. of Math. 123(1986), 11-42.

[7] V. B. Mehta and C. S. Seshadri. Moduli of vector bundles over curves with parabolic structures. Math. Ann. 248 (1980), 205-239.

[8] W. Schmid. Variation of Hodge structure: the singularities of period mapping. Invent. Math. 22 (1973), 211-319.

Department of Mathematical Sciences, University of Tokyo,

Komaba, Meguro, Tokyo, 153-8914, Japan

kawamata@ms.u-tokyo.ac.jp 